\title{
MUC16 provides immune protection by inhibiting synapse formation between NK and ovarian tumor cells
}

\author{
Jennifer AA Gubbels ${ }^{1}$, Mildred Felder ${ }^{1}$, Sachi Horibata', Jennifer A Belisle ${ }^{1}$, Arvinder Kapur ${ }^{1}$, Helen Holden ${ }^{1}$,
} Sarah Petrie ${ }^{1}$, Martine Migneault ${ }^{2}$, Claudine Rancourt ${ }^{2}$, Joseph P Connor ${ }^{1}$, Manish S Patankar ${ }^{{ }^{*}}$

\begin{abstract}
Background: Cancer cells utilize a variety of mechanisms to evade immune detection and attack. Effective immune detection largely relies on the formation of an immune synapse which requires close contact between immune cells and their targets. Here, we show that MUC16, a heavily glycosylated 3-5 million Da mucin expressed on the surface of ovarian tumor cells, inhibits the formation of immune synapses between NK cells and ovarian tumor targets. Our results indicate that MUC16-mediated inhibition of immune synapse formation is an effective mechanism employed by ovarian tumors to evade immune recognition.

Results: Expression of low levels of MUC16 strongly correlated with an increased number of conjugates and activating immune synapses between ovarian tumor cells and primary naïve NK cells. MUC16-knockdown ovarian tumor cells were more susceptible to lysis by primary NK cells than MUC16 expressing controls. This increased lysis was not due to differences in the expression levels of the ligands for the activating receptors DNAM-1 and NKG2D. The NK cell leukemia cell line (NKL), which does not express KIRs but are positive for DNAM-1 and NKG2D, also conjugated and lysed MUC16-knockdown cells more efficiently than MUC16 expressing controls. Tumor cells that survived the NKL challenge expressed higher levels of MUC16 indicating selective lysis of MUC16 low targets. The higher csMUC16 levels on the NKL resistant tumor cells correlated with more protection from lysis as compared to target cells that were never exposed to the effectors.

Conclusion: MUC16, a carrier of the tumor marker CA125, has previously been shown to facilitate ovarian tumor metastasis and inhibits NK cell mediated lysis of tumor targets. Our data now demonstrates that MUC16 expressing ovarian cancer cells are protected from recognition by NK cells. The immune protection provided by MUC16 may lead to selective survival of ovarian cancer cells that are more efficient in metastasizing within the peritoneal cavity and also at overcoming anti-tumor innate immune responses.
\end{abstract}

\section{Introduction}

Ovarian cancer is the deadliest of the gynecological malignancies. Eighty percent of the 14,000 cases of ovarian cancer that are diagnosed each year are of epithelial cell origin. Epithelial ovarian cancer is associated with the formation of a large amount of peritoneal fluid and is extremely metastatic. Immune regulation plays an important role in controlling ovarian tumor growth. Infiltration of $\mathrm{T}$ cells within the tumor is strongly

\footnotetext{
* Correspondence: patankar@wisc.edu
'Department of Obstetrics and Gynecology, University of Wisconsin-Madison,

* Correspondence: patankar@wisc.edu
'Department of Obstetrics and Gynecology, University of Wisconsin-Madison, Madison, USA
}

associated with an increase in 5-year survival of ovarian cancer patients [1]. Primary cancer cells are known to express PVR and nectin, ligands for the activating NK cell receptor DNAM-1 [2,3]. Recognition of these ligands results in lysis of ovarian cancer cells by naïve NK cells present in the systemic circulation.

Ovarian tumors, however, have developed elaborate mechanisms to counter immune recognition and attack. Factors produced by the tumor can alter the expression of important activating molecules on immune cells present in the peritoneal cavity. In one study, a $10-14 \mathrm{kDa}$ protein produced by the tumor cells was shown to downregulate the expression of the key signaling 


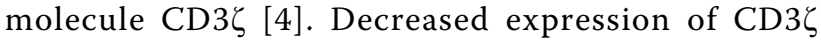
causes impairment of the immune response $[4,5]$. Macrophage migration inhibitory factor (MIF) produced by ovarian tumor cells decreases the transcription and expression of the activating receptor NKG2D on NK cells thereby inhibiting their ability to recognize and lyse ovarian tumor targets [6]. Other NKG2D ligands expressed by ovarian cancer cells include MICA, MICB, and Letal [7-9].

We have studied the effects of one particular factor produced in high quantities by the tumor cells, MUC16, and its effect on the cytolytic function of human NK cells $[10,11]$. MUC16 is a membrane spanning mucin with an average molecular weight between 3-5 million $\mathrm{Da}$ [12]. The high molecular weight of MUC16 is a result of the over 24,000 amino acids that constitute the protein backbone and also the extensive O-linked and $\mathrm{N}$-linked glycosylation of this molecule [12-14]. Ovarian tumors present MUC16 as a Type I membrane glycoprotein on their cell surface. We refer to the cell surface bound mucin as csMUC16. Proteolytic cleavage at a site 50 amino acids upstream of the transmembrane region is hypothesized to result in shedding of the mucin from ovarian tumors $[12,15,16]$. The shed mucin, sMUC16, is present at considerable concentration (5-20 nM) in the peritoneal fluid and also leaks into the systemic circulation. csMUC16 and sMUC16 carry a repeating peptide epitope that has been previously characterized as the ovarian tumor marker CA125 [13,17].

sMUC16 is a potent inhibitor of the cytolytic ability of NK cells [11]. Incubation of NK cells from healthy donors with sMUC16 results in a 40-70\% decrease in surface expression of CD16 [10,11]. Downregulation of CD16, a low affinity Fc receptor, impairs the ability of the peritoneal NK cells to mediate Antibody-Dependent Cell Mediated Cytotoxicity (ADCC) [5,18-22]. Thus sMUC16 directly inhibits the natural cytotoxicity mechanism of NK cells and may also indirectly attenuate ADCC in NK cells of ovarian cancer patients.

To date, csMUC16 has not been studied for its potential role in protecting ovarian tumor cells from immune attack. csMUC16, similar to sMUC16, may directly interact with NK cells and inhibit their ability to lyse tumor targets. Alternatively, csMUC16 may also protect ovarian tumor cells from NK attack by a different mechanism. Mucins are known to possess both adhesive and anti-adhesive properties [23]. csMUC16 acts as an anti-adhesive molecule in the endometrium and must be absent for successful trophoblast adherence to the endometrial wall [24]. MUC16 has also been shown to have a barrier function against pathogen adherence on corneal epithelial cell layers [25-27]. RNA interference of MUC16 expression in human corneal-limbal epithelial cells resulted in increased Staphylococcus aureus adhesion on the corneal epithelial cell layer [25].

Because NK cells form immune synapses with their target cells [28-30], which involve very close cell-cell contact, the presence of an anti-adhesive molecule on the surface of ovarian tumor cells may have significant consequences for tumor cell interactions with NK cells. The formation of these synapses allows NK cells to effectively read the inhibitory or activating ligands on the surface of the target cells. The strength and level of expression of these ligands determines the subsequent action of the NK cell [31]. The inability of NK cells to form these synapses would restrict effector cell activation and subsequent lysis of tumor targets.

Here, we provide evidence that the expression of csMUC16 attenuates the interactions between ovarian cancer cells and NK cells. Our data indicates that NK cells are unable to form immune synapses with the ovarian tumor targets, regardless of the expression of activating or inhibitory ligands on the surface of the tumor cells. This results in another redundant molecular mechanism that epithelial ovarian tumor cells utilize to avoid immune attack.

\section{Results}

NK cells preferentially target csMUC $16^{\text {low }}$ OVCAR-3 cells OVCAR-3 is an established ovarian tumor cell line that expresses csMUC16 and releases sMUC16 into the culture media. This cell line was also used for the cloning of MUC16 [17]. This cell line is therefore a good model to study the function of sMUC16 and csMUC16 [32]. NK cells derived from peripheral blood of healthy donors are unable to efficiently lyse OVCAR-3 cells $[33,34]$. To determine the role of csMUC16 in immune protection we conducted confocal microscopy experiments to analyze the direct interactions between OVCAR-3 cells and NK cells. The OVCAR-3 cell population exhibits a wide range of csMUC16 expression as determined by flow cytometry [35]. We developed an arbitrary scale to categorize OVCAR-3 as csMUC16 ${ }^{\text {low }}$, csMUC16 $6^{\text {medium }}$, and csMUC16 ${ }^{\text {high }}$ based on fluorescent intensity as visualized by microscopy (Figure 1A).

OVCAR-3 cells were incubated with NK cells derived from peripheral blood of healthy donors. Invariably, we observed that NK cells selectively conjugated with csMUC16 ${ }^{\text {low }}$ OVCAR-3 cells as compared to csMUC16medium and csMUC16 ${ }^{\text {high }}$ cells (Figure 1A, B). Quantification of NK cell-OVCAR-3 conjugates clearly showed that the effector cells formed 6-7-fold more conjugates with csMUC16 ${ }^{\text {low }}$ OVCAR-3 cells compared to csMUC16 ${ }^{\text {high }}$ OVCAR-3 (Figure 1C). Intermediate numbers of conjugates were observed between NK cells and OVCAR-3 cells expressing medium levels of csMUC16. 


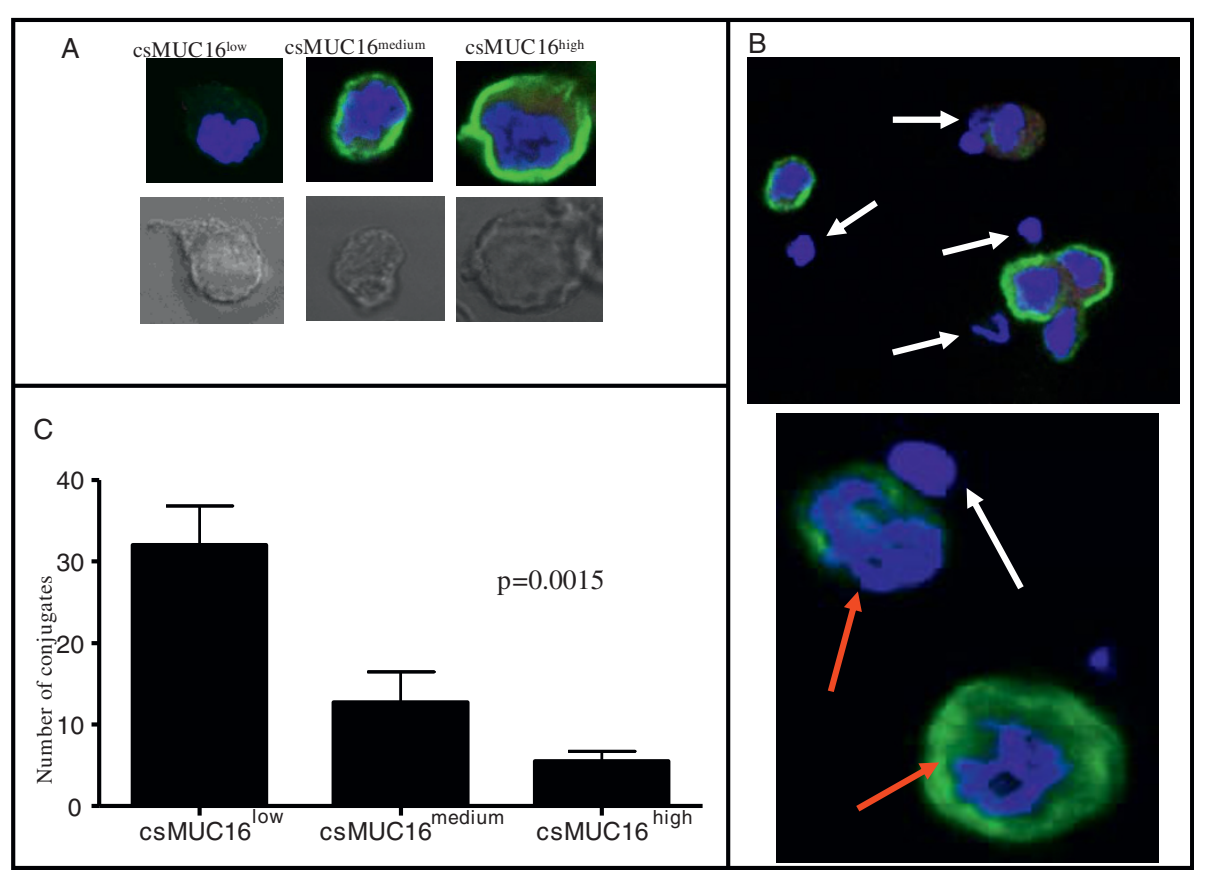

Figure 1 Selective targeting of csMUC16 ${ }^{\text {low }}$ OVCAR-3 cells by NK cells. A, Upper panels show the classification of cSMUC16 low, medium, and high OVCAR-3 cells. This classification was used to determine NK cell conjugation with different subsets of OVCAR-3 cells. The lower panel shows the corresponding bright field image of the cell in the upper panel. $B$, The upper panel is a 60X view of NK cells (white arrows) forming

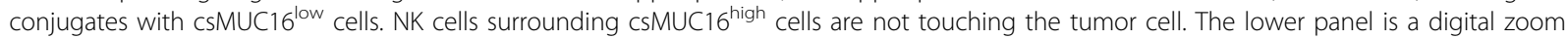
image demonstrating selective conjugation of NK cells (white arrow) with csMUC16 low and not with csMUC16 ${ }^{\text {high }}$ OVCAR-3 cells (red arrows). C, Conjugates of NK cells with low, medium and high csMUC16 expressing cells were quantified by averaging the number of conjugates observed per 50 fields. Data shown is mean of four independent experiments performed with NK cells from separate donors. A one-way ANOVA was performed and the resulting $p$ value $=.0015$.

csMU16 ${ }^{\text {low }}$ cells express comparable levels of DNAM-1 and NKG2D ligands

One potential reason for differential binding between NK cells and csMUC16 low or high expressing OVCAR-3 cells could be that the csMUC16 ${ }^{\text {low }}$ cells were expressing higher levels of ligands of the activating receptors DNAM-1 and NKG2D that have been shown to play an important role in lysing primary ovarian cancer cells $[2,8,9,36]$. To test this hypothesis, expression of DNAM-1 and NKG2D ligands on the OVCAR-3 cells was examined in relation to csMUC16 levels.

DNAM-1-Fc or NKG2D-Fc chimeras were added to OVCAR-3 cells and the cells were gated for high or low csMUC16 expression (Figure 2). OVCAR-3 cells expressing low or high csMUC16 expressed comparable levels of DNAM-1 and NKG2D ligands (Figure 2). These data indicate that differences in expression of DNAM-1 or NKG2D ligands was not a factor in the ability of NK cells to conjugate to OVCAR-3 cells.

\section{MUC16 knock-down OVCAR-3 cells}

The significantly reduced ability of NK cells to bind to csMUC16 ${ }^{\text {high }}$ OVCAR-3 cells suggested a potential role for this mucin in protecting ovarian cancer cells from immune recognition. To investigate this immuno- protective role, we employed MUC16 knock-down cells that were successfully utilized in our recent studies $[35,37]$. The subline csMUC16 ${ }^{\text {neg }}$-OVC expresses an endoplasmic reticulum localized $\mathrm{scFv}$ fragment of the anti-MUC16 antibody VK-8 that prevents expression of csMUC16 and sMUC16. A control subline designated csMUC16 ${ }^{\text {pos }}$-OVC that expresses an scFv fragment of an irrelevant murine IgG has no effect on the expression of csMUC16 and sMUC16 [37]. Expression of csMUC16 on both sublines is shown in Figure 3. The scFvMUC16 complex is rapidly degraded in the proteasomes and the mucin does not accumulate in the cytoplasm of the tumor cells (see additional file 1). The csMUC16 ${ }^{\text {pos }}$ OVC and csMUC16 ${ }^{\text {neg }}$-OVC were negative for MUC4 expression and did not exhibit any major difference in the levels of MUC1 (Figure 3). This is important to note as it has previously been shown that both MUC1 and MUC4 exert anti-adhesive effects on immune synapse formation [38,39].

Expression of DNAM-1 and NKG2D ligands and HLA class I on CsMUC16 ${ }^{\text {pos }}$-OVC and CsMUC $16^{\text {neg }}$-OVC

NKG2D and DNAM-1 ligand expression and presentation of HLA class I antigens on tumor cells are important factors that determine activation or inhibition of 


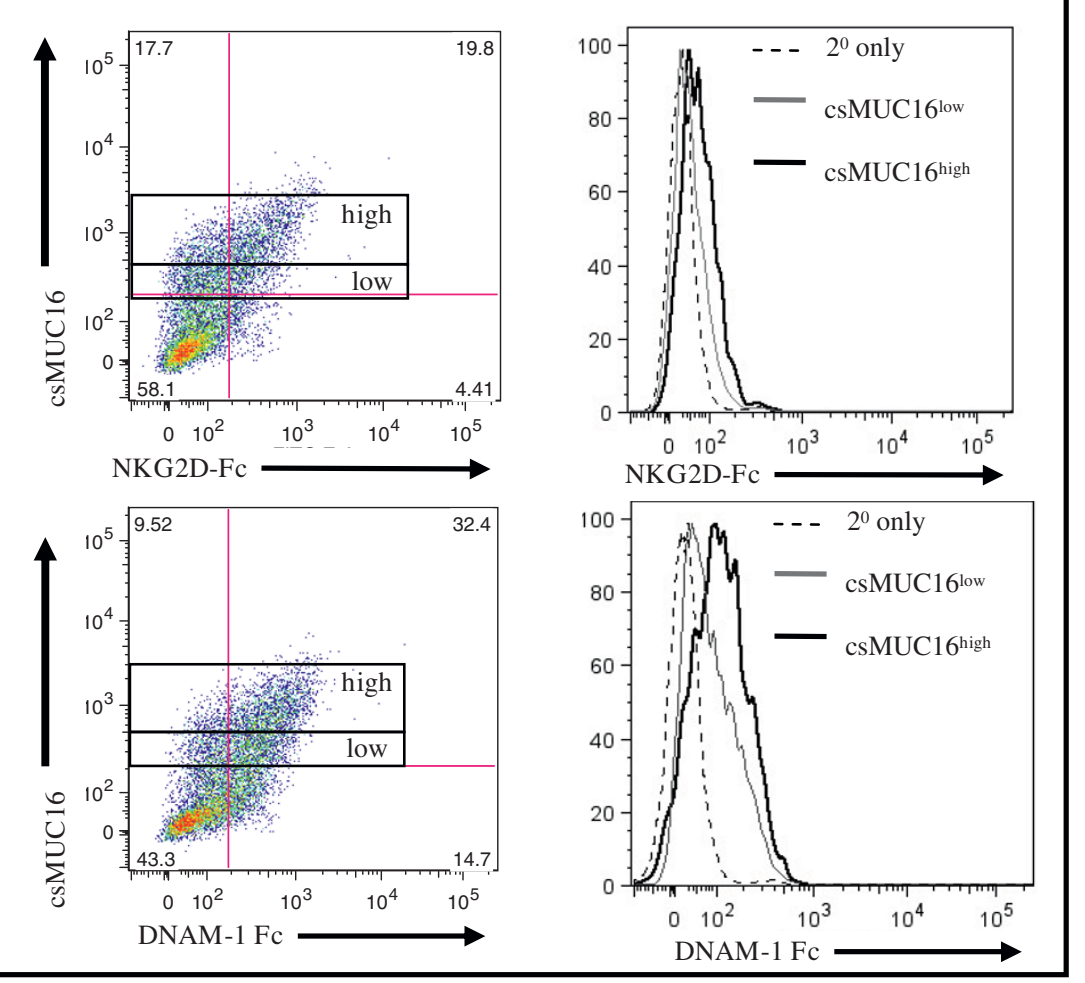

Figure 2 DNAM-1 and NKG2D ligand expression is comparable on csMUC16 ${ }^{\text {high }}$ expressing OVCAR-3 cells. OVCAR-3 cells were stained for csMUC16 expression, and then NKG2D-FC or DNAM-1-FC and an appropriate secondary antibody were added. Cells were initially gated on live single events of tumor cells that were csMUC $16^{\text {low }}$ or $\operatorname{csMUC16^{\text {high}}}$. The binding of NKG2D-FC or DNAM-1 to gated csMUC16 ${ }^{\text {low }}$ (gray line) and $\operatorname{csMUC16^{\text {high}}}$ (black line) is shown in histogram form. Data is representative of three independent experiments.

NK cells. csMUC16 $6^{\text {neg }}-\mathrm{OVC}$ and csMUC16 ${ }^{\text {pos }}-\mathrm{OVC}$ express comparable levels of DNAM-1 and NKG2D ligands, as determined by monitoring the binding of DNAM-1-Fc and NKG2D-Fc chimeras to these two sublines (Figure 3). Similarly comparable levels of MHC class I antigens were expressed by csMUC $16^{\text {neg }}-\mathrm{OVC}$ and $\mathrm{CsMUC} 16^{\text {pos }}$-OVC (Figure 3).

\section{csMUC16 protects csMUC $16^{\text {pos }}$-OVC from NK cell lysis}

HLA class I antigens serve as strong inhibitory ligands of the Killer Immunoglobulin-like Receptors (KIR) of NK cells. Therefore, HLA class I expression protects tumor cells from NK cell attack. However, although comparable levels of HLA class I were expressed on the csMUC16 ${ }^{\text {pos }}$ OVC and csMUC16 ${ }^{\text {neg }}$-OVC the sublines exhibited differential susceptibility to NK cell mediated lysis. Higher protection of the $\operatorname{csMUC} 16^{\text {pos }}$-OVC from NK cell mediated cytolysis as compared to the csMUC16 ${ }^{\text {neg }}-\mathrm{OVC}$ was consistently observed (Figure 4). NK cells used in these assays were obtained from the peripheral blood of four healthy donors. Cytotoxicity assays at 10:1, 5:1 and $1: 1$ effector cell:target cell ratios showed that the csMUC16 ${ }^{\text {neg }}-\mathrm{OVC}$ were lysed at a significantly higher level than the csMUC16 ${ }^{\text {pos }}$-OVC (Figure 4).
NKL cells lack KIR expression and are more effective at killing csMUC16 ${ }^{\text {neg }}-$ OVC

As shown in Figure 3, both the csMUC16 ${ }^{\text {pos }}$-OVC and csMUC16 ${ }^{\text {neg }}$-OVC expressed HLA class I antigens that could contribute to their protection from NK cell mediated lysis. Therefore, another effector cell model was required where the protection provided by HLA class I antigens would not be as pronounced as with the primary NK cells from healthy donors.

The NK cell leukemia cell line, NKL, does not express the prominent KIR (Killer Immunoglobulin-like Receptors) CD158a, CD158b, and CD158e (Figure 5A). These KIRs are inhibitory receptors that bind to HLA class I molecules and abrogate the cytotoxic responses of NK cells [40]. The lack of KIR on NKL cells therefore reduces the inhibitory effect arising out of a major pathway (HLA class I-KIR) displayed by the csMUC16 ${ }^{\text {pos }}$ -

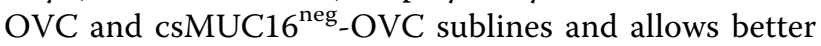
isolation of the immunoprotective properties of csMUC16.

To assess the ability of NKL cells to lyse either subline, flow cytometry based cytotoxicity assays were performed with these cells. There was a 2-3 fold increase in 

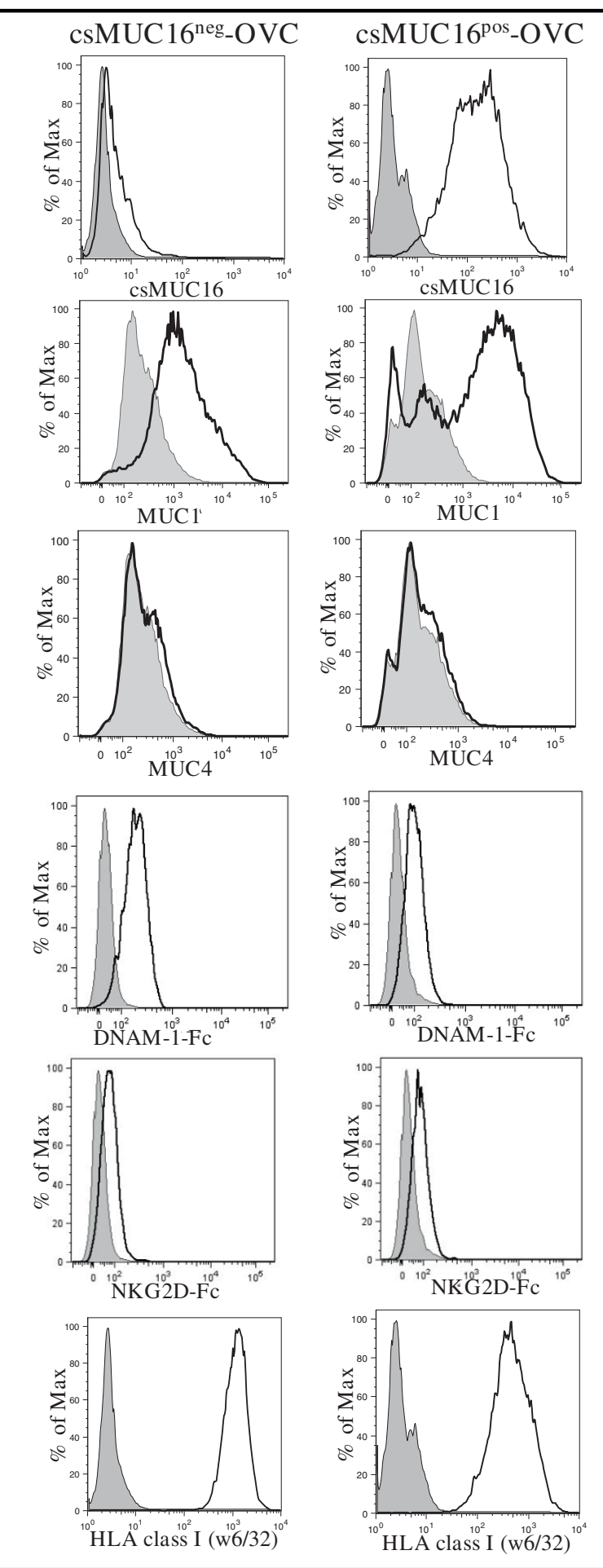

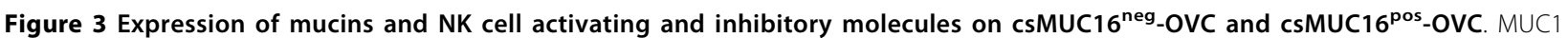
expression on both sublines is similar and MUC4 expression is absent. Expression of DNAM-1 and NKG2D ligands on the sublines was determined by staining with $\mathrm{Fc}$ chimeras $(15 \mu \mathrm{g} / \mathrm{mL})$ of these two receptors followed by labeling with fluorophore conjugated anti-Fc secondary antibody. HLA class I expression on the sublines was determined by labeling with FITC conjugated w6/32. Histograms of live single events are shown in all plots. Data is representative of three independent experiments. Shaded peaks show control cells incubated with only the fluorescently-tagged secondary antibodies. Un-shaded histograms are for cells incubated with the primary antibodies or Fc chimeras and the fluorescently labeled secondary antibodies. 


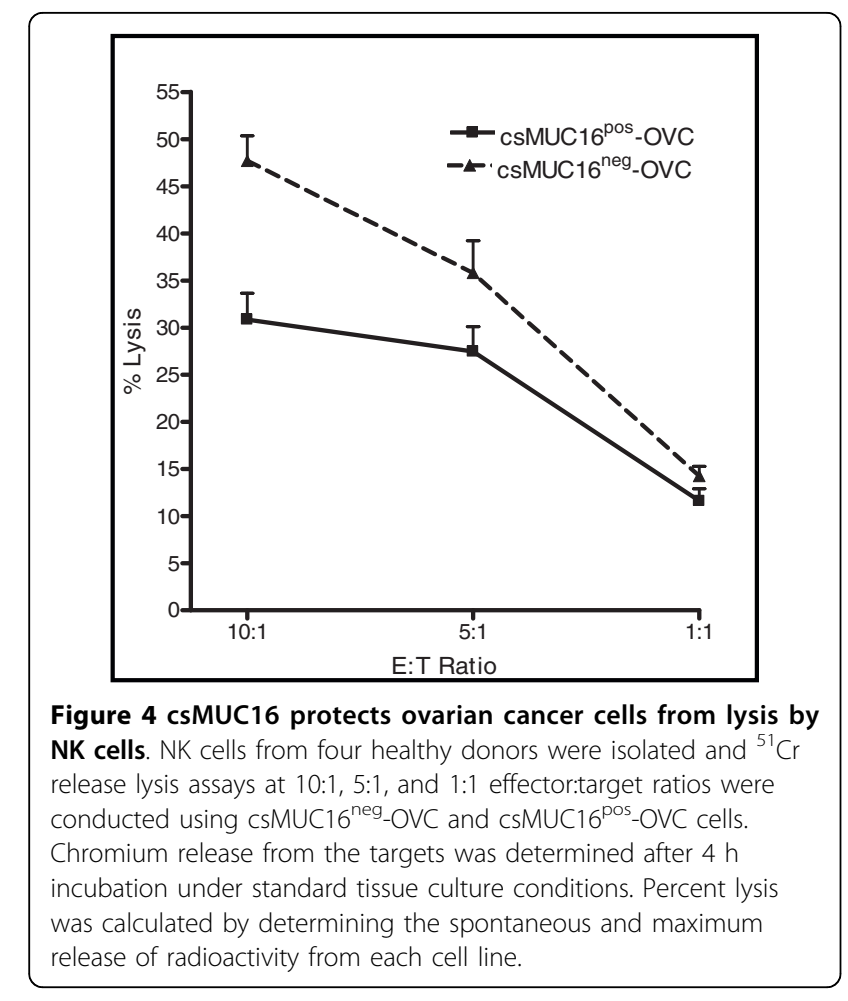

killing when NKLs were paired with csMUC16 ${ }^{\text {neg }}$-OVC compared to csMUC16 $6^{\text {pos }}$-OVC (Figure 5B).

CsMUC16 ${ }^{\text {pos }}$-OVC form low numbers of conjugates with NK and NKL cells

Because NK cells were selectively binding to csMUC16 ${ }^{\text {low }}$ OVCAR-3 cells (Figure 1), we determined if they also exhibited a higher ability to conjugate with csMUC16 $^{\text {neg }}$-OVC. We first quantified doublet formation between fluorescently tagged effector and target cells by flow cytometry. Approximately 1.5 -fold more doublets were observed between NK cells (isolated from three different healthy donors) and csMUC16 ${ }^{\text {neg }}-\mathrm{OVC}$ as compared to csMUC16 $6^{\text {pos }}$-OVC (Figure 6A).

Similar to our observations with NK cells, we were able to demonstrate that $\mathrm{csMUC} 16^{\text {neg }}-\mathrm{OVC}$ formed increased numbers of conjugates in plate-based adhesion assays with NKL cells compared to csMUC16 ${ }^{\text {pos }}$-OVC (Figure 6B). Higher adhesion correlated with 2-3-fold higher killing of the csMUC16 ${ }^{\text {neg }}$-OVC by the NKL cells (Figure 5B).

csMUC16 ${ }^{\text {neg }}-$ OVC cells formed higher numbers of activating immune synapses with NK cells

Conjugation between NK cells and targets results in formation of immune synapses. NK cells form activating immune synapses in order to lyse target cells. We

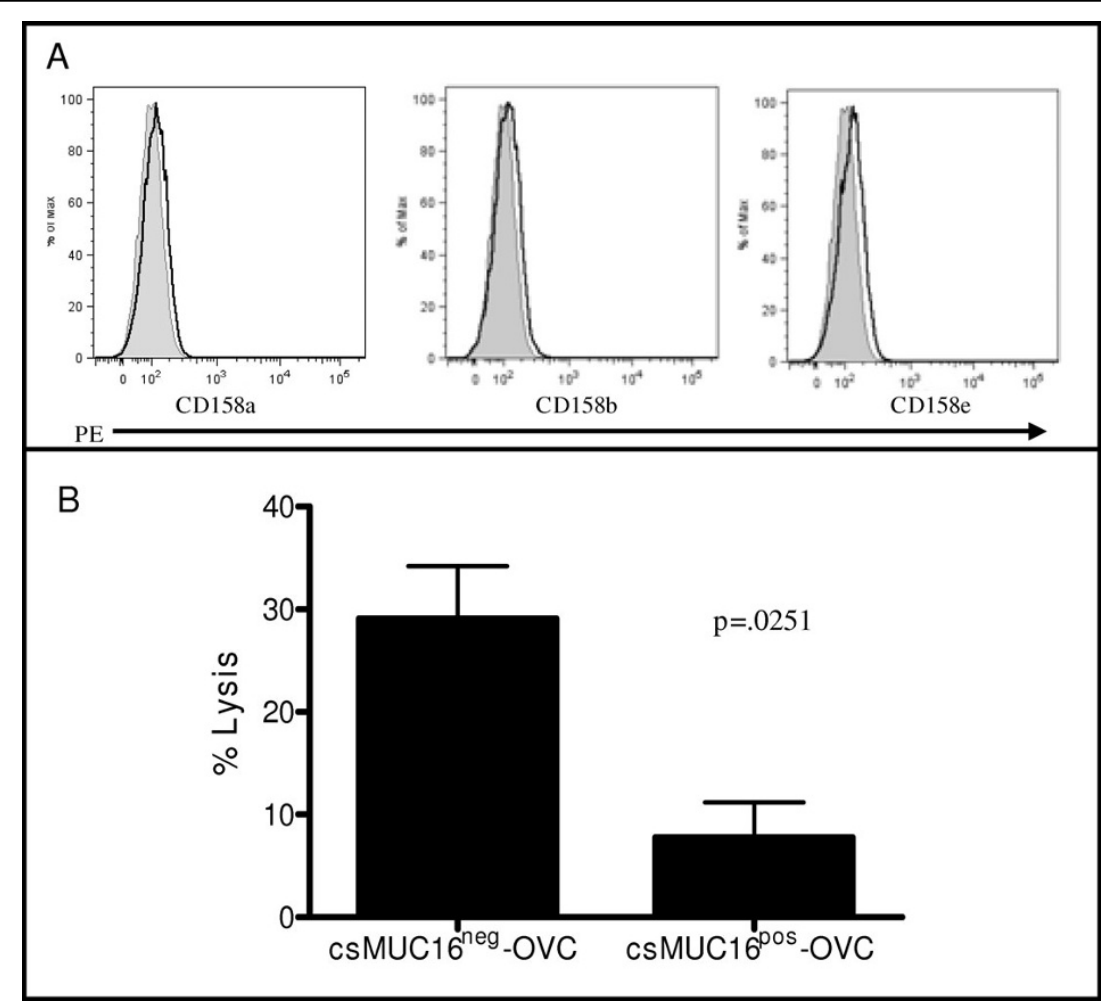

Figure 5 NKLs lack KIRs and exhibit reduced ability to lyse csMUC16 ${ }^{\text {pos }}$-OVC. A Expression of KIR on NKL cells was determined by flow cytometry. B. Flow cytometry based cell lysis assays (24:1 effector:target ratio) were conducted to demonstrate that NKL cells lyse csMUC16 $6^{\text {neg }}$ OVC cells to a greater extent than the CsMUC16 ${ }^{\text {pos }}$-OVC cells. Mean, standard deviation and p-value of three independent measurements is shown. 
therefore determined if the increased conjugation between csMUC16 ${ }^{\text {neg }}$-OVC and NK cells resulted in the formation of activating immune synapses. Activating immune synapses between NK cells and csMUC16 ${ }^{\text {pos }}-\mathrm{OVC}$ and csMUC16 ${ }^{\text {neg }}-\mathrm{OVC}$ were quantified by determining the polarization of LFA-1 or CD2 and F-actin at the interface between the effector and target cells. csMUC16 ${ }^{\text {neg }}-\mathrm{OVC}$ cells formed twice as many activating immune synapses with NK cells compared to csMUC16 ${ }^{\text {pos }}$-OVC (Figure 7). In some cases, multiple NK cells were also found to form simultaneous activating immune synapses with the csMUC16 ${ }^{\text {neg }}-\mathrm{OVC}$ cells (Figure 7B). Although NK cells were found in the vicinity of $\operatorname{csMUC} 16^{\text {pos }}$-OVC, activating immune synapses were not formed (Figure 7B).

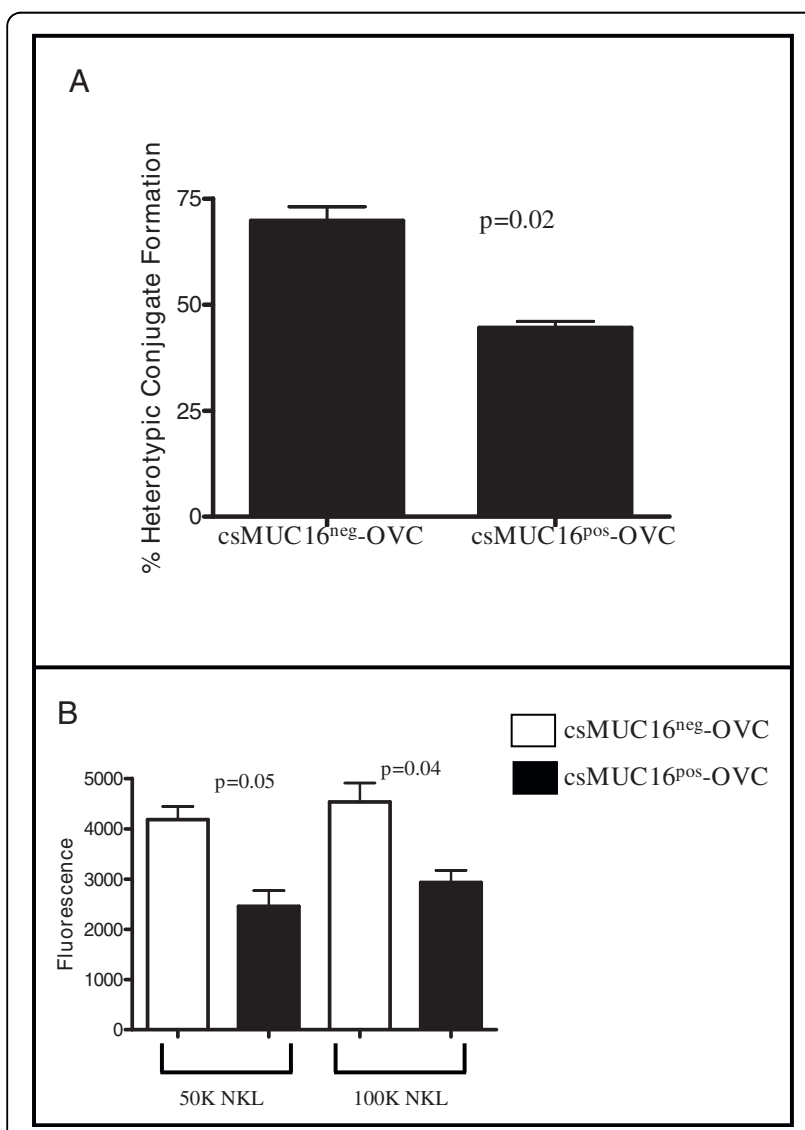

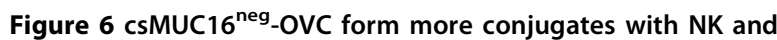
NKL cells than csMUC $16^{\text {pos }}$-OVC. A, NK cells from healthy donors were labeled with CellTracker Green and mixed with CellTracker

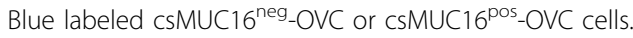

Conjugation between the tumor cells and NK cells was determined by flow cytometry. Mean values of NK cell-tumor cell conjugation obtained from experiments conducted with three healthy donors

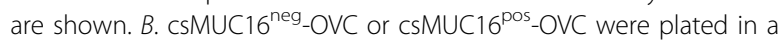
96-well plate and allowed to grow to $70-80 \%$ confluence. Calcein dyed NKLs were added to the plate. After incubation, non-adherent cells were removed by washing and the NKL cells bound to tumor targets were quantified using a fluorescence plate reader. Data for two experiments was combined for a total of 12 replicates.
csMUC16 protects adherent tumor cells from NKLmediated lysis

The ability of NKL cells to selectively lyse csMUC16 ${ }^{\text {neg }}$ OVC and csMUC16 ${ }^{\text {pos }}$-OVC was further evaluated in co-culture experiments. An additional MUC16-knockdown clone (csMUC16 ${ }^{\text {neg }}$-OVC-A) was also used in these experiments. This protocol for the generation of this additional cell line has been reported [37]. The three sublines were plated at equal densities in separate wells. After cells were $70 \%$ confluent, NKL cells were introduced in the cultures and plates were incubated for $24 \mathrm{~h}$ (Figure $8 \mathrm{~A}$ ) or $48 \mathrm{~h}$ (Figure $8 \mathrm{~B}$ ). At the end of the incubation, NKL cells and lysed non-adherent ovarian tumor cells were removed and the remaining adherent colonies and individual cells were manually counted. Very few adherent colonies and cells per observed field remained in the csMUC16 ${ }^{\text {neg }}-\mathrm{OVC} / \mathrm{NKL}$ and csMUC16neg-OVC-A/NKL co-cultures (Figure 8). However, 3-4fold more colonies and cells/field were detected in the csMUC16 ${ }^{\text {pos }}$-OVC/NKL co-cultures (Figure 8).

NKL-resistant csMUC16 ${ }^{\text {pos }}$-OVC express higher levels of csMUC16

Adherent csMUC16 ${ }^{\text {pos }}$-OVC cells that were resistant to two sequential NKL challenges (Figure 8) were designated as csMUC16 ${ }^{\text {pos }}$-OVC-R. The surviving csMUC16pos-OVC-R cells were further cultured to $100 \%$ confluence and csMUC16 expression on these cells was determined by flow cytometry. Compared to csMUC16pos_OVC that were cultured at identical confluence and similar passage number, but had not been exposed to NKL cells, the csMUC16 ${ }^{\text {pos }}$-OVC-R expressed 2-fold higher levels of csMUC16 (Figure 9A).

Based on all of the data obtained in this study we predicted that the csMUC16 ${ }^{\text {pos }}-\mathrm{OVC}-\mathrm{R}$ would be more resistant to killing by effector cells due to the even higher expression of csMUC16. Indeed, when NKL cells were used as effector cells, the csMUC16 ${ }^{\text {pos }}$-OVC-R cells showed a 3-4-fold decreased susceptibility to lysis as compared to the csMUC $16^{\text {pos }}$-OVC that had not been previously subjected to NKL treatment (Figure 9B). These data are consistent with results obtained from the cytotoxicity experiments using csMUC16 ${ }^{\text {pos }}$-OVC and csMUC16 ${ }^{\text {neg }}$-OVC and also support our initial observation that NK cells selectively form decreased numbers of conjugates with OVCAR-3 cells that express higher levels of csMUC16.

\section{Discussion}

An NK cell lyses target cells based on the expression level and strength of activating and inhibitory signals. Expression of HLA class I antigens on ovarian tumor cells acts as a strong signal that abrogates the cytotoxic responses of NK cells by serving as ligands for the inhibitory KIR and LIR receptors. In addition, signal 


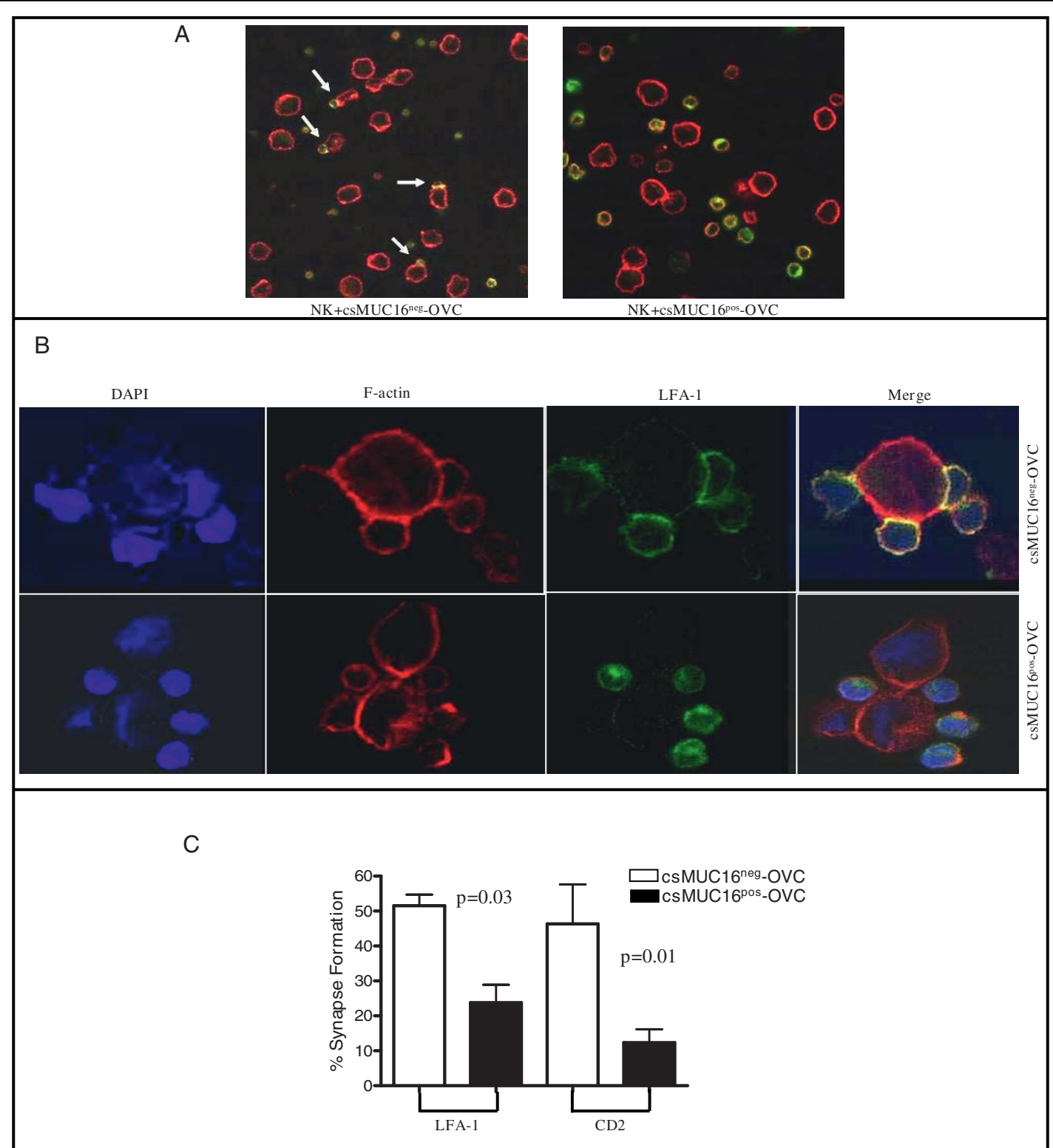

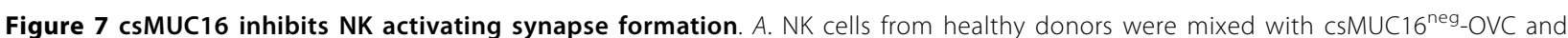
CsMUC16 ${ }^{\text {pos }}$-OVC cells and confocal microscopy was conducted using 10x magnification. White arrows indicate NK cells bound to tumor cells and some forming activating immune synapses. B. Immune synapses between the NK cells and csMUC16 ${ }^{\text {neg_OVC }}$ at $40 \times$ magnification are shown. NK cells are present in the vicinity of CsMUC16 $6^{\text {pos }}$-OVC cells but form significantly less conjugates and immune synapses. C. Immune synapses between NK cells from three healthy donors and the CsMUC16 ${ }^{\text {neg-OVC }}$ or the CSMUC16 ${ }^{\text {pos }}-$ OVC cells were quantified by counting conjugates that showed polarization of LFA-1 and CD2 divided by the total number of conjugates (defined as two cells in contact).

peptides of HLA class I antigens bind to the non-polymorphic HLA-E molecules [41]. The signal peptideHLA-E complex is presented on the cell surface where it serves as a ligand for the inhibitory CD94/NKG2A receptor complex expressed on NK and other immune cells [42-44]. On the other hand, ovarian tumor cells also express ligands for the activating receptors NKG2D and DNAM-1 [2] that make them susceptible to NK cell mediated lysis.
Recognition of inhibitory or activating ligands requires NK cells to form close contacts with tumor cells. The formation of such cell-cell interactions is dependent upon the molecular landscape of the cell surface on both cell types. While adhesive molecules are required to formalize contacts between the cancer and effector cells, anti-adhesive molecules may hinder proper recognition of the tumors by NK cells. This study identifies csMUC16 as an inhibitor of NK cell/tumor cell contact 


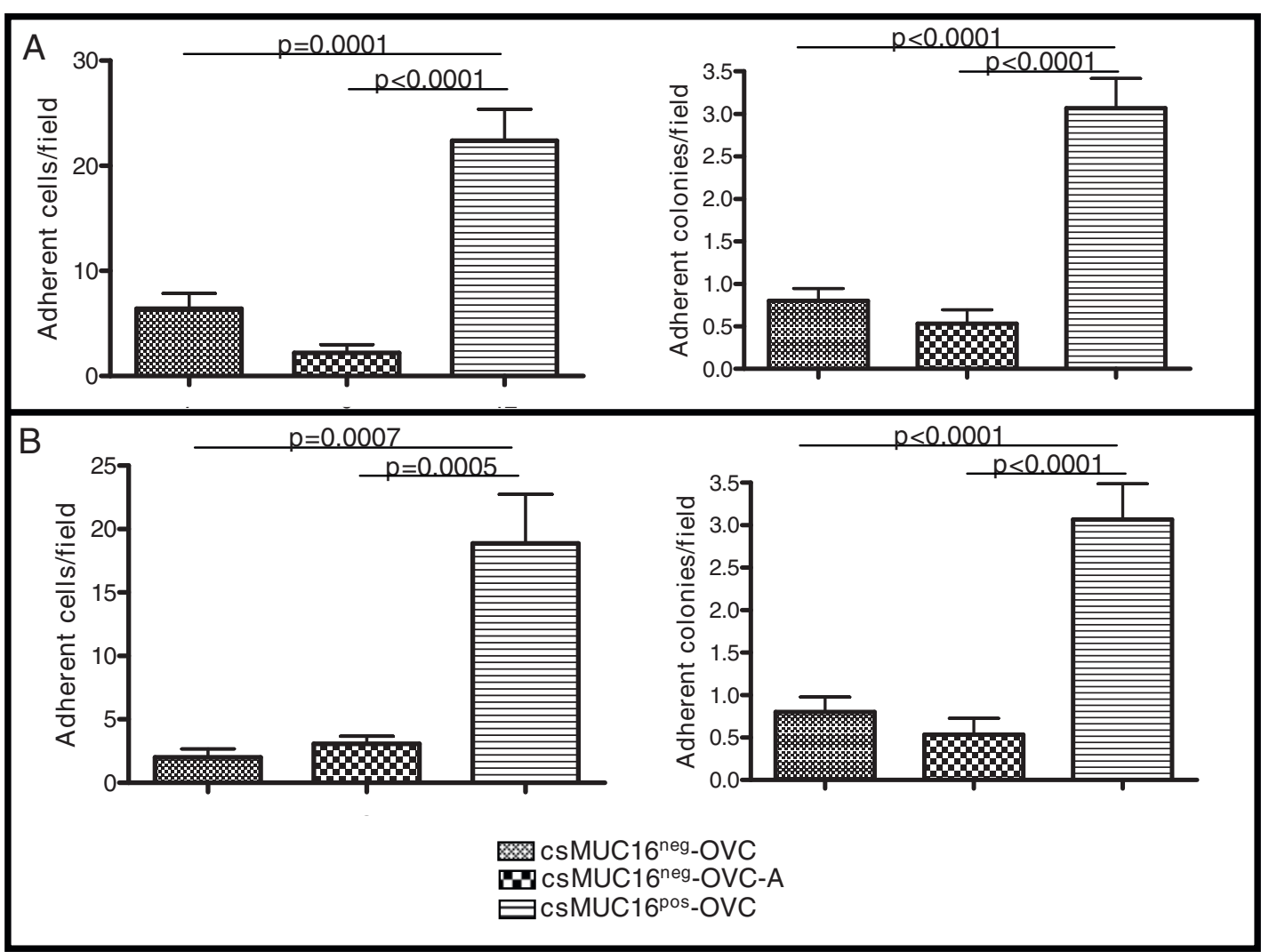

Figure 8 csMUC16 shields ovarian cancer cells from NKL mediated lysis. A. NKL cells were added to confluent cultures of csMUC16 ${ }^{\text {neg-OVC }}$ or csMUC16 ${ }^{\text {pos }}$-OVC cells at a 1:1 effector:target ratio. After incubation for A, $24 \mathrm{~h}$ and $B, 48 \mathrm{~h}$ the number of adherent colonies and adherent cells surviving the NKL challenge were counted by microscopic examination of five random fields. This experiment was repeated in triplicate and mean values ( $n=15$ fields) and standard deviation are shown.

thereby providing a mechanism for ovarian tumor cells to evade immune recognition.

NK cells isolated from peripheral blood of healthy donors preferentially form conjugates with OVCAR-3 cells that expressed lower levels of csMUC16 (Figure 1). We determined that the high degree of targeting of the csMUC $16^{\text {low }}$ OVCAR-3 cells was not due to higher expression of NKG2D or DNAM-1 ligands. The role of csMUC16 in attenuating NK cell recognition of ovarian cancer cells was delineated by using the csMUC16 knockdown OVCAR-3 subline, csMUC16 ${ }^{\text {neg }}$-OVC. The csMUC16 ${ }^{\text {neg }}-\mathrm{OVC}$ and the matching control subline csMUC16 ${ }^{\text {pos }}$-OVC are similar in their expression of NKG2D and DNAM-1 ligands. HLA class I levels were slightly elevated on csMUC16 ${ }^{\text {neg }}-\mathrm{OVC}$ cells compared to $\mathrm{csMUC1} 6^{\text {pos }}$-OVC. These observations would suggest that $\mathrm{csMUC} 16^{\text {neg }}$-OVC would be better protected from NK cell killing as compared to csMUC16 ${ }^{\text {pos }}$-OVC. To the contrary, primary NK cells lyse the csMUC16 ${ }^{\text {neg }}$ OVC to a significantly greater extent than the csMUC16 ${ }^{\text {pos }}$-OVC. Similar effect was observed when the $\mathrm{KIR}^{\text {neg }} \mathrm{NKL}$ cells were used as effectors. Because of the lack of KIR, the expression of HLA class I on the targets has a markedly reduced inhibitory effect on the cytolytic capacity of the NKL cells. Therefore, lower amount of lysis of the csMUC16 $6^{\text {pos }}$-OVC observed in the NKL experiments is primarily due to the protection provided by csMUC16.

We have shown previously that sMUC16 binds specifically to a subset of NK cells in epithelial ovarian cancer patients. Our current data indicates that the receptor for sMUC16 is the inhibitory receptor Siglec-9 (Belisle et al manuscript in preparation). The binding of csMUC16 to Siglec-9 could be a mechanism by which csMUC16 is causing inhibition of NK cells. We have shown using cytotoxicity assays that NKL cells are also inhibited by MUC16 present on csMUC16 ${ }^{\text {pos }}$-OVC cells. This is significant because NKLs are Siglec-9 negative and do not bind to sMUC16 (data not shown), indicating Siglec-9csMUC16 binding may not play a role in the observed inhibition of cytotoxicity.

An alternate explanation for NK cell protection afforded by csMUC16 lies in the extraordinary biochemical properties of the molecule itself. Mucins are known 
to exhibit both adhesive and anti-adhesive properties. In cancer, the loss of apical expression of these large molecules and the subsequent distribution over the entire cell membrane has consequences for cell-cell interactions [45]. The large size of the molecules as well as the extensive $\mathrm{O}$-glycosylation present on mucins contributes to their anti-adhesive properties. MUC16 contains a large number of core 2 type O-glycans [14]. The overexpression of core 2 type $\mathrm{O}$-glycans on $\mathrm{T}$ cells has been implicated in disrupting cell-cell interactions between $\mathrm{B}$ and $\mathrm{T}$ cells, preventing cross-talk between the cells and impairing the humoral immune response [46].

MUC16 has a protein backbone of approximately 24,000 amino acids [12] and a high degree of glycosylation, indicating that it may extend a considerable distance out from the surface of the cell. Mucins such as MUC1, MUC4 and MUC16 have semi-flexible rod-like structures extending outwards from the cell surface $[45,47]$. It is estimated that a 28 -mer peptide with Oglycan chains has a linear length of approximately $7 \mathrm{~nm}$ [47]. Based on these calculations, csMUC16, with an approximately 24,000 amino acid protein backbone, can be expected to extend up to $1-5 \mu \mathrm{m}$ from the surface of ovarian tumor cells. The NK synaptic cleft requires apposition of the effector and the target cells over a distance of 10-55 nm [48]. Thus, ovarian cancer cells may specifically utilize the very large and heavily glycosylated csMUC16 molecule to prevent the conjugation and eventual binding to NK cells.

Other large molecules have been shown to interfere with cell-cell contact because of the bulky nature of their extracellular domains. CD43, which is abundantly expressed on T cells and is heavily glycosylated, is removed from the immune synapse before synapse formation between T cells and APCs [49]. Other researchers have shown that dendritic cells induce the polarization of MUC1 on T cells to sites opposing the T cell-dendritic cell interface [39]. Carraway and colleagues have conclusively demonstrated that MUC4, another large molecular weight mucin, shields cancer cells from lysis by the lymphokine activated killer cells due to its anti-adhesive properties [38]. As the expression of both MUC1 and MUC4 were similar on csMUC16 ${ }^{\text {pos }}-\mathrm{OVC}$ and csMUC16 ${ }^{\text {neg }}-\mathrm{OVC}$, these mucins did not contribute to the differences in conjugation and lysis that were seen between these two sublines.

The targeting of the csMUC16 ${ }^{\text {low }}$ ovarian tumor cells by NK cells may result in the selective survival of csMUC16 ${ }^{\text {high }}$ cancer cells. Such a selective increase in csMUC16 ${ }^{\text {high }}$ ovarian cancer cells is supported by our observation that csMUC16 ${ }^{\text {pos }}$-OVC that were resistant to NKL attack expressed a higher level of csMUC16 (Figure 9A). Higher expression of csMUC16 on the surviving cancer cells may not only allow them to withstand NK cell killing, as demonstrated in Figure 9B, but also provide them an avenue to do so while expressing lower levels of HLA and thereby reducing their vulnerability to $\mathrm{T}$ cells.

Our data may represent an example of an immunoediting mechanism that allows progression and proliferation of ovarian tumors. NK cells may selectively eliminate csMUC16 ${ }^{\text {low }}$ cells. The csMUC16 ${ }^{\text {high }}$ cells surviving NK cell attack not only have the advantage of effectively evading immune attack but can also utilize csMUC16 to adhere to mesothelial cells at various sites within the peritoneal cavity [35]. The surviving csMUC16 ${ }^{\text {high }}$ ovarian tumor cells may also be expected to shed higher amounts of sMUC16. We have now demonstrated that sMUC16 binds Siglec-9, an inhibitory receptor present on cytolytic NK cells in addition to subsets of B cells and a majority of monocytes (Belisle et al manuscript in preparation). Selective immunoediting of csMUC16 $6^{\text {high }}$ ovarian tumors may therefore lead to tumors that have a higher potential to withstand immune attack and also to metastasize and proliferate in the peritoneal environment.

\section{Conclusion}

We have conclusively shown that the expression of csMUC16 blocks the conjugation step of immune synapse formation of NK cells with ovarian cancer targets. This blockage prevents NK cell lysis of cancer cells. Immune protection afforded by csMUC16 should therefore be considered as another important mechanism that promotes tumor growth.

\section{Materials and methods \\ Reagents and effector cell isolation}

Unless otherwise stated, all chemicals were purchased from either Sigma or Fisher. NK cells were isolated from healthy donors using the RosetteSep (Stem Cell Technologies) NK Cell Isolation Kit protocol. Purity of the isolated NK cells was between 80 and $90 \%$ based on flow cytometry using anti-CD3, CD16, and CD56 antibodies. Antibodies against MUC1, CD2, CD3, CD16, CD56, CD158a, CD158b, CD158e, and HLA Class I (w6/32), were from BD Biosciences. Anti-LFA-1 and anti-MUC4 antibodies were from eBiosciences and Zymed Laboratories, respectively. OVCAR-3 cells were obtained from ATCC (Rockville, Maryland). OVCAR-3 csMUC16 knockdown sublines csMUC16 $6^{\text {neg }}-\mathrm{OVC}$ and csMUC16 ${ }^{\text {neg }}$-OVC-A and control subline csMUC16 ${ }^{\text {pos }}$ OVC were obtained as described in our recent studies $[37,35]$.

\section{Slide preparation and analysis for confocal microscopy}

NK-target synapse experiments were conducted according to previously established protocols $[30,50,51]$. OVCAR-3, csMUC16 $6^{\text {pos }}-$ OVC, and csMUC16 ${ }^{\text {neg }}-$ OVC 


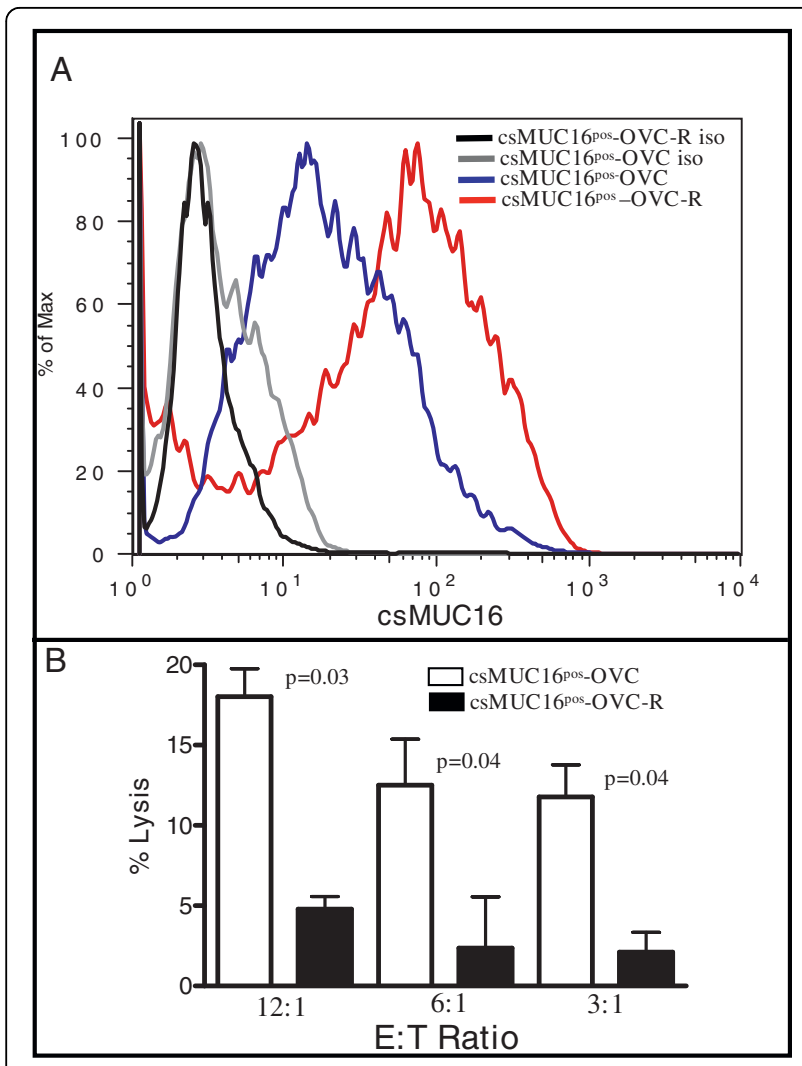

Figure 9 Tumor cells surviving NKL challenge express higher levels of csMUC16. A. CsMUC $16^{\text {pos }}-\mathrm{OVC}$ cells not treated with NKL or those surviving NKL treatment (CSMUC16 ${ }^{\text {pos }}-\mathrm{OVC}-\mathrm{R}$ ) were analyzed for csMUC16 expression by flow cytometry. Data is representative of three independent experiments. $B_{1}$ csMUC $16^{\text {pos }}$ OVC and CsMUC $16^{\text {pos }}$-OVC-R were labeled with ${ }^{51} \mathrm{Cr}$. Cytotoxity assays were performed by using NKL cells as effectors. After $4 \mathrm{~h}$ coincubation, released radioactivity was measured. Each bar is mean of four independent assays.

cells were cultured in the conditions prescribed for OVCAR-3 cells by ATCC. Target cells $\left(7.5 \times 10^{4}\right.$ cells $)$ were mixed with NK cells at a 1:1 ratio in a total volume of $400 \mu \mathrm{l}$. The cells were centrifuged for $5 \mathrm{~min}$ utes at $100 \times \mathrm{g}$ and $\mathrm{I}$ incubated at $37^{\circ} \mathrm{C}$ at $5 \% \mathrm{CO}_{2}$ for 25 minutes. The media was aspirated and cells were resuspended in PBS without calcium and magnesium and placed on poly-L-lysine coated coverslips. All subsequent procedures were performed at room temperature. Cells were fixed with 3\% paraformaldehyde (Polysciences, Inc.) for $15 \mathrm{~min}$, washed twice with $150 \mathrm{mM}$ glycine, permeabilized with $0.1 \%$ Triton-X for $4 \mathrm{~min}$, washed with PBS, and blocked overnight with PBS containing $5 \%$ goat serum.

Cells were stained with murine antibodies against LFA-1, CD2, or MUC16 (VK8 antibody kindly provided by Dr. Beatrice Yin) in 5\% goat serum for $30 \mathrm{~min}$. Coverslips were washed with $1 \mathrm{~mL}$ PBS, stained with a cocktail of goat anti-mouse FITC (Jackson
ImmunoResearch), and rhodamine conjugated phalloidin (Invitrogen) for $30 \mathrm{~min}$. Cells were washed with PBS, dipped in deionoized water, and mounted with DAPI containing mounting media (Invitrogen). After drying overnight, cells were visualized using the Bio-Rad Radiance 2100 MP Rainbow confocal microscope. Fifty conjugates between NK and tumor targets were counted on each coverslip. Conjugates were defined as an effector (NK cell) and target (cancer cell line) in contact with one another. Each conjugate was scored for polarization of the activating synapse markers LFA-1 and F-actin or $\mathrm{CD} 2$ and F-actin. Polarization was determined using two criteria, both of which had to be met to be defined as a synapse. These criteria were as follows: if greater than $70 \%$ of the fluorescence (of CD2 and F-actin or LFA-1 and F-actin) was noted to be at the interface between target and effector cell, and if there was a noticeable "flattening" of the membranes at the contact interface, than the synapse was deemed an activating synapse. Percent synapse formation was determined as: (number of conjugates showing polarization of activating synapse markers/total conjugates $\times 100$ ).

\section{Flow cytometry antibody staining}

For flow cytometric analysis, cells were washed two times in PBS containing $1 \%$ BSA by centrifugation at $300 \times \mathrm{g}$ for 10 minutes at $4^{\circ} \mathrm{C}$. Cells were labeled with primary and secondary antibodies for $30 \mathrm{~min}$ on ice, and washed with PBS containing 1\% BSA in between antibody staining and prior to flow cytometry. In the DNAM-1-Fc and NKG2D-Fc binding experiments, OVCAR-3, csMUC $16^{\text {pos }}$-OVC or csMUC $16^{\text {neg }}-$ OVC were blocked with Goat IgG (BD Biosciences) for 15 min., washed, and $15 \mu \mathrm{g} / \mathrm{mL}$ of either DNAM-Fc or NKG2D-Fc (R\&D Systems) was added for $30 \mathrm{~min}$ on ice. Goat anti-human-Fc FITC (Jackson ImmunoResearch) was added for $30 \mathrm{~min}$ and washed. To determine mucin and HLA class I expression cells were treated with the primary antibodies. Where appropriate, cells were stained with goat anti-mouse PE (1:100, Jackson ImmunoResearch). Immediately before data acquisition on an LSRII (Beckton Dickinson) flow cytometer, the viability indicator PI or DAPI were added to each sample. Automatic compensation was applied. Flowjo software (v. 4.6.1, TreeStar) was used for analysis of the raw flow cytometry data, and comparison and statistical analysis (student t-test) of the data was done using GraphPad Prism software (v. 4, GraphPad Software, Inc.).

\section{Chromium-based cytotoxicity assays}

csMUC16 ${ }^{\text {neg }}-\mathrm{OVC}$, csMUC16 ${ }^{\text {pos }}-\mathrm{OVC}$, or $\mathrm{CsMUC} 16^{\text {pos }}$ OVC-R were labeled with ${ }^{51} \mathrm{Cr}$ in suspension. The targets were mixed with NK cells isolated from 4 healthy donors in 96-well plates at different effector:target ratios. After $4 \mathrm{~h}$ incubation, ${ }^{51} \mathrm{Cr}$ released from the targets was measured and percent lysis was determined as 
described previously [52]. Comparison and statistical analysis (student t-test) of the data was done using GraphPad Prism software (v. 4, GraphPad Software, Inc.).

\section{Cytotoxicity Assays of adherent csMUC $16^{\text {neg }}$-OVC and csMUC16 ${ }^{\text {pos }}$-OVC cells}

CellTracker Green (Invitrogen) labeled NKL cells were added to wells of 6-well plates that contained confluent csMUC16 ${ }^{\text {neg }}-\mathrm{OVC}$ or csMUC16 ${ }^{\text {pos }}-$ OVC cells. After 24 $\mathrm{h}$ incubation, the media and the adherent cells were harvested using trypsin-EDTA, transferred to a tube, stained with PI, and analyzed for percentage of live target cells by flow cytometry on a BD LSR-II instrument. Comparison and statistical analysis (student t-test) of the data was done using GraphPad Prism software (v. 4, GraphPad Software, Inc.).

Lysis of adherent csMUC16 ${ }^{\text {neg }}-\mathrm{OVC}$ and $\operatorname{csMUC} 16^{\text {pos }}$ OVC cells by NKL cells was also measured by counting the number of surviving adherent tumor targets. These experiments were set up similarly to the flow cytometry based cytotoxicity assays except that NKL cells were not stained. Following co-culture, the floating NKL cells and dead tumor cells were removed by gentle washing and the number of adherent (considered live) cells and colonies were counted on an inverted microscope. The cell counts from three independent experiments were pooled and the averages $(n=15)$ were plotted. csMUC16 ${ }^{\text {pos }}$ OVC cells that survived NKL challenge were further cultured to confluence and re-challenged with NKL cells. Tumor cells surviving this second NKL treatment were harvested and analyzed by flow cytometry for amount of csMUC16 expression.

\section{Flow cytometry conjugate formation assay}

Freshly isolated naïve NK cells were labeled with $0.5 \mu \mathrm{M}$ CellTracker Red (Invitrogen) for 25 minutes at $37^{\circ} \mathrm{C}$ in $5 \% \mathrm{CO}_{2}$. Concurrently, csMUC16 ${ }^{\text {neg }}-\mathrm{OVC}$ and csMUC1 $6^{\text {pos }}$-OVC cells were trypsinized, washed, and dyed with $1.25 \mathrm{pM}$ CellTracker Green (Invitrogen) in the same conditions. After washing off excess dye with PBS, cells were resuspended in PBS containing 1\% BSA solution. NK cells were placed in flow tubes with either csMUC $16^{\text {neg }}-$ OVC or csMUC $16^{\text {pos }}-$ OVC cells at a $1: 1$ ratio and centrifuged for 2 minutes at $100 \times \mathrm{g}$. Tubes were incubated for 25 minutes at room temperature, and vortexing was avoided. Immediately before data acquisition on an LSRII (Beckton Dickinson) flow cytometer, viability indicator DAPI (BD Biosciences) was added to each sample. Comparison and statistical analysis (student t-test) of the data was done using GraphPad Prism software (v. 4, GraphPad Software, Inc.).

\section{Plate adhesion assays}

csMUC16 ${ }^{\text {neg }}-\mathrm{OVC}$ and $\mathrm{csMUC} 16^{\text {pos }}-\mathrm{OVC}$ were plated at 100,000 cells/well of a 96-well plate and allowed to grow for 48 hours to confluence. On the day of the assay, NKL (natural killer leukemia) cells were dyed with calcein AM (1 ug/mL, Invitrogen) in 1\% PBS-BSA for $30 \mathrm{~min}$ at $37^{\circ} \mathrm{C}$, washed, and resuspended in media containing FBS at a concentration of 50,000 or 100,000 NK cells/50 uL. Fifty $\mu \mathrm{L}$ of dyed NKLs were added to each well containing the previously plated $\operatorname{csMUC} 16^{\text {neg }}$ OVC or csMUC16 ${ }^{\text {pos }}$-OVC. The plate was centrifuged at $400 \times \mathrm{g}$ for 5 minutes and placed in the incubator for 25 minutes. The plate was washed gently with $1 \%$ PBSBSA three times to remove non-adherent cells and then read using the Victor V-3 plate reader (Perkin Elmer). Comparison and statistical analysis (student t-test) of the data was done using GraphPad Prism software (v. 4, GraphPad Software, Inc.).

Additional file 1: MUC16 is not detected in the lysates of

csMUC16 ${ }^{\text {neg }}-$ OVC. 1. Lysates of OVCAR-3, CSMUC $16^{\text {pos }}-$ OVC, and

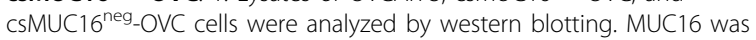
detected by using VK-8 (anti-CA125) as the primary antibody. Actin was used as loading control.

Click here for file

[http://www.biomedcentral.com/content/supplementary/1476-4598-9-11S1.PPT]

\section{Acknowledgements}

We thank Dr Beatrice Yin for providing us the anti-MUC16 antibody VK-8 and Dr. Alexander Rakhmilevich for critical reading of the manuscript. We especially thank Dr. Ilene Gipson for critically reading this manuscript and for insightful comments. Funding for this research was provided by grants from the Department of Defense (\#W81XWH-04-1-0102), Ovarian Cancer Research Fund (UW/UWM.05), UW School of Medicine and Public Health Wisconsin Partnership Program, a charitable donation from Jean McKenzie, start-up funds from the Department of Obstetrics and Gynecology to MSP, and a T32 training grant (JAAG). We are deeply grateful to Kathy Schell for her advice and help and acknowledge the support provided by the University of Wisconsin Comprehensive Cancer Centers Flow Cytometry facility which is supported by a core grant (CA14520) from the National Institutes of Health.

\section{Author details}

'Department of Obstetrics and Gynecology, University of Wisconsin-Madison, Madison, USA. 'Department of Microbiology and Infectiology, Universite de Sherbrooke, Sherbrooke, Canada.

\section{Authors' contributions}

JAAG conducted the majority of the experiments and helped in designing experiments, data analysis, and preparing the manuscript. MF and JAB conducted some of the flow cytometry experiments. SH conducted the cell binding experiments. $\mathrm{HH}$ and SP assisted in maintaining the cell lines and isolation of blood samples. MM and CR developed the MUC16 knockdown cell lines. AK assisted in monitoring MUC16 expression by the knockdown cell lines. JPC assisted in designing the experiments and data interpretation. MSP is the corresponding author and helped in study design, data interpretation and preparation of the manuscript. All authors read and approved the final manuscript.

\section{Competing interests}

The authors declare that they have no competing interests.

Received: 10 August 2009

Accepted: 20 January 2010 Published: 20 January 2010 


\section{References}

1. Zhang L, Conejo-Garcia JR, Katsaros D, Gimotty PA, Massobrio M, Regnani G Makrigiannakis A, Gray H, Schlienger K, Liebman MN, Rubin SC, Coukos G: Intratumoral T cells, recurrence, and survival in epithelial ovarian cancer. N Engl J Med 2003, 348:203-213.

2. Carlsten M, Bjorkstrom NK, Norell H, Bryceson $Y$, van Hall T, Baumann BC, Hanson M, Schedvins K, Kiessling R, Ljunggren HG, Malmberg KJ: DNAX accessory molecule-1 mediated recognition of freshly isolated ovarian carcinoma by resting natural killer cells. Cancer Res 2007, 67:1317-1325.

3. Carlsten M, Malmberg KJ, Ljunggren HG: Natural killer cell-mediated lysis of freshly isolated human tumor cells. Int J Cancer 2009, 124:757-762.

4. Taylor DD, Gercel-Taylor C, Lyons KS, Stanson J, Whiteside TL: T-cell apoptosis and suppression of T-cell receptor/CD3-zeta by Fas ligandcontaining membrane vesicles shed from ovarian tumors. Clin Cancer Res 2003, 9:5113-5119.

5. Lai $P$, Rabinowich $H$, Crowley-Nowick PA, Bell MC, Mantovani G, Whiteside TL: Alterations in expression and function of signaltransducing proteins in tumor-associated T and natural killer cells in patients with ovarian carcinoma. Clin Cancer Res 1996, 2:161-173.

6. Krockenberger M, Dombrowski Y, Weidler C, Ossadnik M, Honig A, Hausler S, Voigt H, Becker JC, Leng L, Steinle A, Weller M, Bucala R, Dietl J, Wischhusen J: Macrophage migration inhibitory factor contributes to the immune escape of ovarian cancer by down-regulating NKG2D. J Immunol 2008, 180:7338-7348.

7. Li K, Mandai M, Hamanishi J, Matsumura N, Suzuki A, Yagi H, Yamaguchi K, Baba T, Fujii S, Konishi I: Clinical significance of the NKG2D ligands, MICA/ $B$ and ULBP2 in ovarian cancer: high expression of ULBP2 is an indicator of poor prognosis. Cancer Immunol Immunother 2009, 58:641-652.

8. Groh V, Wu J, Yee C, Spies T: Tumour-derived soluble MIC ligands impair expression of NKG2D and T-cell activation. Nature 2002, 419:734-738.

9. Conejo-Garcia JR, Benencia F, Courreges MC, Gimotty PA, Khang E, Buckanovich RJ, Frauwirth KA, Zhang L, Katsaros D, Thompson CB, et al: Ovarian carcinoma expresses the NKG2D ligand Letal and promotes the survival and expansion of CD28- antitumor T cells. Cancer Res 2004 64:2175-2182.

10. Belisle JA, Gubbels JA, Raphael CA, Migneault M, Rancourt C, Connor JP, Patankar MS: Peritoneal natural killer cells from epithelial ovarian cancer patients show an altered phenotype and bind to the tumour marker MUC16 (CA125). Immunology 2007, 122:418-429.

11. Patankar MS, Yu J, Morrison JC, Belisle JA, Lattanzio FA, Deng Y, Wong NK, Morris HR, Dell A, Clark GF: Potent suppression of natural killer cell response mediated by the ovarian tumor marker CA125. Gynecol Oncol 2005, 99:704-713.

12. O'Brien TJ, Beard JB, Underwood LJ, Dennis RA, Santin AD, York L: The CA 125 gene: an extracellular superstructure dominated by repeat sequences. Tumour Biol 2001, 22:348-366.

13. Yin BW, Lloyd KO: Molecular cloning of the ca125 ovarian cancer antigen. identification as a new mucin, muc16. J Biol Chem 2001, 276:27371-27375.

14. Kui Wong $N$, Easton RL, Panico M, Sutton-Smith M, Morrison JC, Lattanzio FA, Morris HR, Clark GF, Dell A, Patankar MS: Characterization of the oligosaccharides associated with the human ovarian tumor marker CA125. J Biol Chem 2003, 278:28619-28634.

15. Davis HM, Zurawski VR Jr, Bast RC Jr, Klug TL: Characterization of the CA 125 antigen associated with human epithelial ovarian carcinomas. Cancer Res 1986, 46:6143-6148.

16. Hattrup CL, Gendler SJ: Structure and Function of the Cell Surface (Tethered) Mucins. Annu Rev Physiol 2007, 70:431-457.

17. Yin BW, Dnistrian A, Lloyd KO: Ovarian cancer antigen CA125 is encoded by the MUC16 mucin gene. Int J Cancer 2002, 98:737-740.

18. Lotzova E, Savary CA, Freedman RS, Bowen JM: Natural killer cell cytotoxic potential of patients with ovarian carcinoma and its modulation with virus-modified tumor cell extract. Cancer Immunol Immunother 1984, 17:124-129.

19. Connor JP, Felder M, Hank J, Harter J, Gan J, Gillies SD, Sondel P: Ex vivo evaluation of anti-EpCAM immunocytokine huKS-IL2 in ovarian cancer. $J$ Immunother 2004, 27:211-219.

20. Mantovani A, Allavena P, Sessa C, Bolis G, Mangioni C: Natural killer activity of lymphoid cells isolated from human ascitic ovarian tumors. Int $\mathrm{J}$ Cancer 1980, 25:573-582.

21. Allavena P, Zanaboni F, Rossini S, Merendino A, Bonazzi C, Vassena L, Mangioni C, Mantovani A: Lymphokine-activated killer activity of tumor- associated and peripheral blood lymphocytes isolated from patients with ascites ovarian tumors. J Natl Cancer Inst 1986, 77:863-868.

22. Melioli G, Ferrari I, Casartelli G, Ragni N: Lymphocytes isolated from the peritoneal fluid of women with advanced ovarian carcinoma differ significantly from autologous peripheral blood lymphocytes. Gynecol Oncol 1993, 48:301-307.

23. Hollingsworth MA, Swanson BJ: Mucins in cancer: protection and control of the cell surface. Nat Rev Cancer 2004, 4:45-60.

24. Gipson IK, Blalock T, Tisdale A, Spurr-Michaud S, Allcorn S, Stavreus-Evers A, Gemzell K: MUC16 is Lost from the Uterodome (Pinopode) Surface of the Receptive Human Endometrium: In Vitro Evidence That MUC16 Is a Barrier to Trophoblast Adherence. Biol Reprod 2007, 78:134-142.

25. Blalock TD, Spurr-Michaud SJ, Tisdale AS, Heimer SR, Gilmore MS, Ramesh V, Gipson IK: Functions of MUC16 in corneal epithelial cells. Invest Ophthalmol Vis Sci 2007, 48:4509-4518.

26. Blalock TD, Spurr-Michaud SJ, Tisdale AS, Gipson IK: Release of membraneassociated mucins from ocular surface epithelia. Invest Ophthalmol Vis Sci 2008, 49:1864-1871.

27. Argueso P, Guzman-Aranguez A, Mantelli F, Cao Z, Ricciuto J, Panjwani N: Association of cell surface mucins with galectin-3 contributes to the ocular surface epithelial barrier. J Biol Chem 2009, 284:23037-23045.

28. Davis DM, Chiu I, Fassett M, Cohen GB, Mandelboim O, Strominger JL: The human natural killer cell immune synapse. Proc Natl Acad Sci USA 1999, 96:15062-15067.

29. Krzewski K, Strominger JL: The killer's kiss: the many functions of NK cell immunological synapses. Curr Opin Cell Biol 2008, 20:597-605.

30. Orange JS, Harris KE, Andzelm MM, Valter MM, Geha RS, Strominger JL: The mature activating natural killer cell immunologic synapse is formed in distinct stages. Proc Natl Acad Sci USA 2003, 100:14151-14156.

31. Lanier LL: Natural killer cell receptor signaling. Curr Opin Immunol 2003, 15:308-314.

32. Lloyd $\mathrm{KO}$, Yin BW, Kudryashov V: Isolation and characterization of ovarian cancer antigen CA 125 using a new monoclonal antibody (VK-8): identification as a mucin-type molecule. Int J Cancer 1997, 71:842-850.

33. Fuchshuber PR, Lotzova E: Differential oncolytic effect of NK-enriched subsets in long-term interleukin-2 cultures. Lymphokine Cytokine Res 1992, 11:271-276.

34. Fuchshuber PR, Lotzova E, Pollock RE: Antitumor activity, growth, and phenotype of long-term IL-2 cultures of human NK and T lymphocytes. Lymphokine Cytokine Res 1991, 10:51-59.

35. Gubbels JA, Belisle J, Onda M, Rancourt C, Migneault M, Ho M, Bera TK, Connor J, Sathyanarayana BK, Lee B, Pastan I, Patankar MS: MesothelinMUC16 binding is a high affinity, N-glycan dependent interaction that facilitates peritoneal metastasis of ovarian tumors. Mol Cancer 2006, 5:50.

36. Wang $H$, Yang $D, X u$ W, Wang $Y$, Ruan Z, Zhao $T$, Han J, Wu Y: Tumorderived soluble MICs impair CD3(+)CD56(+) NKT-like cell cytotoxicity in cancer patients. Immunol Lett 2008, 120:65-71.

37. Boivin M, Lane D, Piche A, Rancourt C: CA125 (MUC16) tumor antigen selectively modulates the sensitivity of ovarian cancer cells to genotoxic drug-induced apoptosis. Gynecol Oncol 2009, 115:407-413.

38. Komatsu M, Yee L, Carraway KL: Overexpression of sialomucin complex, a rat homologue of MUC4, inhibits tumor killing by lymphokine-activated killer cells. Cancer Res 1999, 59:2229-2236.

39. Vasir B, Avigan D, Wu Z, Crawford K, Turnquist S, Ren J, Kufe D: Dendritic cells induce MUC1 expression and polarization on human T cells by an IL-7-dependent mechanism. J Immunol 2005, 174:2376-2386.

40. Moretta L, Moretta A: Killer immunoglobulin-like receptors. Curr Opin Immunol 2004, 16:626-633.

41. Braud VN, Allan DS, O'Callaghan CA, Soderstrom K, Andrea AD, Ogg GS, Lazetic S, Young NT, Bell Jl, Phillips JH, Lanier LL, McMichael AJ: HLA-E binds to natural killer cell receptors CD94/NKG2A, B, and C. Nature 1998, 391:795-799.

42. Llano M, Lee N, Navarro F, Garcia P, Albar JP, Geraghty DE, Lopez-Botet M: HLA-E-bound peptides influence recognition by inhibitory and triggering CD94/NKG2 receptors: preferential response to an HLA-Gderived nonamer. Eur J Immunol 1998, 28:2854-2863.

43. Lee $N$, Llano M, Carretero M, Ishitani A, Navarro F, Lopez-Botet M, Geraghty DE: HLA-E is a major ligand for the natural killer inhibitory receptor CD94/NKG2A. Proc Natl Acad Sci USA 1998, 95:5199-5204.

44. Lee N, Goodlett DR, Ishitani A, Marquardt H, Geraghty DE: HLA-E surface expression depends on binding of TAP-dependent peptides derived 
from certain HLA class I signal sequences. J Immunol 1998, 160:49514960.

45. Hilkens J, Ligtenberg MJ, Vos HL, Litvinov SV: Cell membrane-associated mucins and their adhesion-modulating property. Trends Biochem Sci 1992, 17:359-363.

46. Tsuboi S, Fukuda M: Overexpression of branched O-linked oligosaccharides on T cell surface glycoproteins impairs humoral immune responses in transgenic mice. J Biol Chem 1998, 273:3068030687.

47. Jentoft N: Why are proteins O-glycosylated?. Trends Biochem Sci 1990, 15:291-294.

48. McCann FE, Vanherberghen B, Eleme K, Carlin LM, Newsam RJ, Goulding D, Davis DM: The size of the synaptic cleft and distinct distributions of filamentous actin, ezrin, CD43, and CD45 at activating and inhibitory human NK cell immune synapses. J Immunol 2003, 170:2862-2870.

49. Ilani T, Khanna C, Zhou M, Veenstra TD, Bretscher A: Immune synapse formation requires ZAP-70 recruitment by ezrin and CD43 removal by moesin. J Cell Biol 2007, 179:733-746.

50. Orange JS, Ramesh N, Remold-O'Donnell E, Sasahara Y, Koopman L, Byrne M, Bonilla FA, Rosen FS, Geha RS, Strominger JL: Wiskott-Aldrich syndrome protein is required for NK cell cytotoxicity and colocalizes with actin to NK cell-activating immunologic synapses. Proc Natl Acad Sci USA 2002, 99:11351-11356.

51. Standeven LJ, Carlin LM, Borszcz P, Davis DM, Burshtyn DN: The actin cytoskeleton controls the efficiency of killer Ig-like receptor accumulation at inhibitory NK cell immune synapses. J Immunol 2004, 173:5617-5625.

52. Colligan JE, Kruisbeck AM, Marguiles DH, Shevac EM, Strober W: Measurement of cytolytic activity of NKLAK 1996.

doi:10.1186/1476-4598-9-11

Cite this article as: Gubbels et al: MUC16 provides immune protection by inhibiting synapse formation between NK and ovarian tumor cells. Molecular Cancer 2010 9:11.

Publish with Biomed Central and every scientist can read your work free of charge

"BioMed Central will be the most significant development for disseminating the results of biomedical research in our lifetime. "

Sir Paul Nurse, Cancer Research UK

Your research papers will be:

- available free of charge to the entire biomedical community

- peer reviewed and published immediately upon acceptance

- cited in PubMed and archived on PubMed Central

- yours - you keep the copyright 